\title{
Biliary tract cancer: current challenges and future prospects
}

This article was published in the following Dove Press journal:

Cancer Management and Research

\section{Michele Ghidini' \\ Claudio Pizzo' \\ Andrea Botticelli ${ }^{2}$ \\ Jens Claus Hahne ${ }^{3}$ \\ Rodolfo Passalacqua' \\ Gianluca Tomasello' \\ Fausto Petrelli ${ }^{4}$}

'Department of Oncology, Azienda Socio Sanitaria Territoriale

of Cremona, Cremona, Italy;

${ }^{2}$ Department of Clinical and

Molecular Medicine, Sant'Andrea

Hospital, Rome, Italy; ${ }^{3}$ Division of

Molecular Pathology, The Institute of

Cancer Research, Sutton, Surrey, UK;

${ }^{4}$ Department of Oncology, Operative

Unit of Oncology, Azienda Socio

Sanitaria Territoriale of Bergamo

Ovest, Treviglio, Bergamo, Italy
Correspondence: Fausto Petrelli Department of Oncology, Operative Unit of Oncology, ASST of Bergamo Ovest, Piazzale Ospedale I, 24047 Treviglio (BG), Italy

Tel +39363342 4420

$\mathrm{Fax}+393633424380$

Email faupe@libero.it
Purpose: Incidence and mortality of biliary tract carcinoma (BTC) are increasing, especially in South America and Asia. Such a disease often bears a dismal prognosis because of diagnosis occurring at late stages and for the frequent relapses after surgery. The aims of this review were to summarize the state of the art of the treatment of BTC and give a view at possible future prospects linked with molecular profiling, immunotherapy, and targeted therapies.

Design: We conducted a systematic literature search using MEDLINE and the 2018 ASCO Meeting abstract databases to identify published clinical trials, translational series, and meeting abstracts. All significant papers and abstracts available to date were included.

Results: For resected BTC, thanks to the BILCAP study, adjuvant chemotherapy (CT) with capecitabine should be regarded as the new standard of care. For locally advanced inoperable and metastatic diseases, the use of chemoradiotherapy and radioembolization has not been supported by any randomized Phase III study. The standard of care remains the combination of CT with gemcitabine and cisplatin, as reported by the ABC- 02 trial. All targeted therapies have failed to improve the survival outcomes, either in combination with CT or as single agents and are not recommended in the treatment of BTC. Whole-exome sequencing and molecular profiling have helped in identifying genetic signatures typical of different BTC subtypes. With this support, new trials with targeted agents and immunotherapy have been designed, and results are awaited. Conclusion: BTC still remains a disease with very few treatment options. Different BTC subtypes own peculiar gene mutations and pathways alterations. Therefore, molecular profiling may be the only key to enable new tailored strategies with targeted agents and immunotherapy. Keywords: surgery, cholangiocarcinoma, adjuvant treatment, first-line treatment, targeted therapies, molecular profiling

\section{Introduction}

Biliary tract carcinoma (BTC) defines a group of rare gastrointestinal tumors including gallbladder cancers (GBCs) and cholangiocarcinomas (CCAs), further divided into intrahepatic cholangiocarcinomas (iCCAs) and extrahepatic cholangiocarcinomas (eCCAs). Extrahepatic disease may be split into perihilar (Klatskin's tumor) and distal CCA. ${ }^{1}$

BTC is also histologically classified into small-duct and large-duct types according to the histological and molecular classification. Small-duct iCCAs often develop against the background of chronic hepatitis or liver cirrhosis. Differently, large-duct cancers may be associated with chronic cholangiopathies (intrahepatic stones and primary sclerosing cholangitis) or premalignant lesions called biliary intraepithelial neoplasia 
(BilIN), suggesting a multistep carcinogenetic process. This classification is also associated with different carcinogenetic processes. Genetic alterations in the dehydrogenase 1 and 2 genes (IDH1/2), BAP1, and FGFR2 are mostly restricted to small-duct iCCAs, whereas mutations in KRAS and SMAD4 are more commonly observed in large-duct cancers. ${ }^{2}$

Incidence and mortality of BTC are increasing, especially in South America and Asia. ${ }^{3}$ Its frequency is higher in patients aged between 50 and 70 years with a 1.5 times higher prevalence for CCAs in men $^{4}$ and GBCs in women (two to six times more). ${ }^{5}$ Usually, BTC is paucisintomatic and characterized by weight loss, fever, and sometimes jaundice and pain. The disease rapidly brings the patient to cachexia and inanition, leading to a quick deterioration of the performance status. ${ }^{6}$ Unfortunately, less than $35 \%$ of patients are diagnosed with early (resectable) disease. ${ }^{7}$ Although surgery represents the only curative approach for localized disease, relapse rate is common. ${ }^{8}$ Radical surgery with adequate lymphadenectomy remains the only potentially curative treatment for early stage BTC. ${ }^{9}$

Most of the new cases $(60 \%-70 \%)$ of BTC are diagnosed at an advanced stage, where treatment options are palliative and mainly based on chemotherapy (CT). ${ }^{10}$ Patients with advanced BTC have a poor prognosis with a median overall survival (mOS) commonly not exceeding 12 months. The activity of second and further lines of treatment is limited. Owing to worsening performance status after first line, best supportive care is often the natural choice after disease progression. $^{7}$

In this review, we summarized the state of the art of the treatment of BTC. Additionally, we tried to give a view at the updates linked with genetic landscape, targeted therapies, and immunotherapy. Surgical and transplant procedures were not included in this review.

\section{Resectable disease}

\section{Adjuvant treatment (AT)}

Owing to the high rate (nearly $50 \%$ ) of local and distance relapse after radical surgery, ${ }^{11}$ AT should be considered after radical resection of early stage BTC. ${ }^{9}$

A systemic review and meta-analysis included 20 studies and supported the use of AT after surgery, with a major benefit obtained from postoperative CT (OR 0.39) and chemoradiotherapy (CTRT; OR 0.61) with respect to radiotherapy (RT, OR 0.98, $P=0.02$ ). Patients who had a resection with positive margins (R1, OR $0.49, P=0.004$ ) and nodal positive disease (OR 0.36, $P=0.002$ ) benefited the most from AT, whereas there was no significant gain in OS given by AT in the whole population included (pooled OR 0.74, $P=0.06) .^{7}$ When CTRT is used, the recommended dose of RT is 45 Gy with concurrent 5-fluorouracil (5-FU) or capecitabine (CAPE). ${ }^{9}$ Among these studies, only one had a randomized Phase III design and tested adjuvant CT with mitomycin C and 5-FU vs surgery alone. This trial included 139 cases of CCAs and 140 GBCs. Five-year OS and disease-free survival in the AT group were higher than those in the control group (respectively, 26\% vs $14.4 \%, P=0.0367$ for OS and $20.3 \%$ vs $11.6 \%, P=0.0210$ for median relapse-free survival [mRFS]) in the GBC cohort, whereas no significant difference was shown for CCAs. ${ }^{12}$ Another recent meta-analysis including only studies of adjuvant $\mathrm{CT}$ reported a significant benefit in weighted mean OS of 4.3 months $(P=0.014)$ obtained with postoperative treatment with a risk of death reduction of $41 \%$ (HR 0.59, $P<0.001) .{ }^{13}$ An Asian randomized Phase III trial of adjuvant gemcitabine (GEM) vs observation in resected BTC showed no significant differences in $\operatorname{mOS}(62.3 \mathrm{vs}$ 63.8 months, respectively, $P=0.964)$ and mRFS (36.0 vs 39.9 months, $P=0.693$ ). There was no survival difference between the two groups in subsets stratified by lymph node and resection margin status. ${ }^{14}$

Recently, two Phase III randomized trials of AT have been completed, and a third one is ongoing (Table 1). The multicenter French PRODIGE 12-ACCORD 18 compared observation after surgery with gemcitabine and oxaliplatin (GEMOX) adjuvant CT. Although feasible and not associated with a deterioration in quality of life, adjuvant GEMOX was not associated with a prolonged RFS with respect to observation, with an mRFS of 30.4 for CT vs 22 months for control, $P=0.31$. The 4 -year RFS was $39.3 \%$ vs $33.2 \% .{ }^{15}$ On the opposite, the British Phase III BILCAP study compared AT with CAPE to observation after radical surgery. By considering intention-to-treat analysis, a significant difference in OS between AT and control was not shown (mOS 51 vs 36 months, HR $0.8, P=0.097)$. The difference became statistically significant only after performing a sensitivity analysis with adjustment for nodal status, grade of disease, and gender (HR $0.71, P<0.01$ ). Even with per-protocol analysis, mOS was significantly higher in the CAPE group (53 vs 36 months, $P=0.028) .{ }^{16}$ Subgroup analyses revealed an improvement in survival with AT for R0 resections (HR 0.73), but the entity of benefit was inferior in R1 population (HR 0.90). Perihilar CCAs did not benefit from CAPE therapy (HR 1.08), whereas the OS advantage was present in the eCCA cohort (HR 0.70). CAPE was active both in node-positive disease and nodenegative disease; however, the first group had worse OS with a 2 -year OS of $50 \%$ compared with $80 \%$ of node-negative 
Table I Randomized Phase III trials of adjuvant CT in resected BTC

\begin{tabular}{|c|c|c|c|c|}
\hline \multirow[t]{2}{*}{ Study/authors } & \multirow{2}{*}{$\begin{array}{l}\text { Therapeutic } \\
\text { regimen }\end{array}$} & \multirow{2}{*}{$\begin{array}{l}\text { Number of } \\
\text { patients }\end{array}$} & \multicolumn{2}{|l|}{ Endpoints } \\
\hline & & & Primary & Secondary \\
\hline Takada et al"I & $\begin{array}{l}\text { Mitomycin } \mathrm{C}+ \\
5-\mathrm{FU} \text { vs obs }\end{array}$ & 508 & 5-year OS (\%), 26 vs I4.4* & 5-year DFS (\%), $20.3 \%$ vs II.6\%* \\
\hline $\begin{array}{l}\text { Prodige } 12-A C C O R D \mid 8, \\
\text { Edeline et } \mathrm{al}^{15}\end{array}$ & GEMOX vs Obs & 196 & mRFS (months), 30.4 vs 22.0 & $\begin{array}{l}\text { Global HrQoL, } 70.8 \text { vs } 83.3 \text { (at } 12 \text { months) and } \\
75.0 \text { vs } 83.3 \text { (at } 24 \text { months) }\end{array}$ \\
\hline BILCAP, Primrose et $\mathrm{a}^{16}$ & CAPE vs obs & 447 & mRFS (months), 25 vs I8* & mOS (months), $5 \mathrm{I}$ vs $36^{*}$ \\
\hline ACTICCA-I trial, Stein et $\mathrm{al}^{18}$ & GEMCIS vs obs & 440 & Ongoing & Ongoing \\
\hline
\end{tabular}

Note: *Statistically significant.

Abbreviations: 5-FU, 5-flurouracil; BTC, biliary tract carcinoma; CAPE, capecitabine; CT, chemotherapy; DFS, disease-free survival; GEMCIS, gemcitabine and cisplatin; GEMOX, gemcitabine and oxaliplatin; HrQoL, health-related quality of life; mOS, median overall survival; mRFS, median relapse-free survival; obs, observation; OS, overall survival.

disease. AT was more active in men (HR 0.70) and less in women (HR 0.93)..$^{17}$

The ongoing ACTICCA-1 trial will probably add further insights in the AT setting. This trial is including resected CCAs and GBCs and will compare AT with gemcitabine and cisplatin (GEMCIS) for 24 weeks with observation only. The primary endpoint of the study is RFS. The estimated primary completion date is April 2019. ${ }^{18}$

Owing to the results of the BILCAP study, adjuvant CT with CAPE was proposed as the new standard of care in the AT of resected BTC.

\section{Advanced inoperable and metastatic disease Liver-directed therapies}

CTRT has been considered a possible option in the treatment of locally advanced and nonresectable BTC with survival rates between 9 and 14 months. ${ }^{9}$ However, the only randomized experience available showed the inferiority of CTRT compared to CT. This Phase II randomized study compared 50 Grays RT in addition to 5-FU and cisplatin (CTRT) with gemcitabine and oxaliplatin (GEMOX) $\mathrm{CT}$ alone in the treatment of nonresectable locally advanced BTC. The trial did not reach its completion due to the slow recruitment. In fact, only 32 patients were randomized. Median PFS (mPFS) and OS were longer in the CT arm compared to those in the CTRT arm (11.0 and 19.9 months in the CT arm vs 5.8 and 13.5 months in the combined arm). HRs were 0.65 for PFS and 0.69 for OS. ${ }^{19}$ Conversely, a retrospective analysis of iCCAs treated with high doses of RT showed a significant improvement in local control $(P=0.009)$ and $\mathrm{OS}(P=0.004)$ when a median biologic equivalent dose $>80.5$ Grays was used..$^{20}$ Yttrium-90 radioembolization represents another possible liver-directed treatment in nonresectable iCCAs, showing similar results to first-line $\mathrm{CT}$ in terms of responses and survival outcomes. However, no randomized prospective studies testing this interventional procedure are currently available. In a retrospective series, mPFS was 20 months when $\mathrm{CT}$ was given concomitantly to radioembolization and mOS after local treatment was not reached, with $46 \%$ of patients obtaining a downstaging and undergoing surgery. ${ }^{21}$ A systematic review and pooled analysis of 12 studies of radioembolization reported a weighted mOS of 15.5 months, with a partial response in $28 \%$ of cases and stable disease in $54 \%$ of patients at 3 months after treatment. ${ }^{22}$

\section{First-line CT}

The standard of care in first-line treatment of advanced BTC is still represented by combination of CT with gemcitabine and cisplatin (GEMCIS; Table 2). Such a regimen was tested in the Phase III randomized ABC-02 trial, which enrolled 410 patients who were assigned to doublet treatment or GEM monotherapy. mOS in the experimental arm was 11.7 vs 8.1 months (HR $0.64, P<0.001)$. mPFS was 8 months with the doublet and 5 months with GEM alone $(P<0.001)$. In addition, GEMCIS determined a better tumor control, with a rate of $81.4 \%$ vs $71.8 \%$ for GEM $(P=0.049){ }^{23}$ GEMCIS were also compared to GEM and S- 1 in the Phase III randomized Asian JCOG1113, FUGA-BT trial (Table 2). This was a noninferiority (NI) trial. After randomizing 354 patients, mOS was 13.4 for standard treatment vs 15.1 for GEM and S-1 (HR 0.95 and $P=0.046$ for NI). mPFS was 5.8 months for GEMCIS vs 6.8 months for the experimental arm (HR 0.86). Because of the reduced amount of gastrointestinal toxicity compared with GEMCIS and the possibility to avoid hydration, GEM and S-1 combination was considered one of the options in the treatment of advanced BTC in Asia. ${ }^{24}$ 
Table 2 Phase II and III trials with at least 50 patients included in first- and second-line treatment of advanced BTC

\begin{tabular}{|c|c|c|c|c|c|c|}
\hline \multirow[t]{2}{*}{ Study/authors } & \multirow[t]{2}{*}{ Phase/type } & \multirow{2}{*}{$\begin{array}{l}\text { Number of } \\
\text { patients }\end{array}$} & \multirow[t]{2}{*}{ Therapeutic regimen } & \multicolumn{3}{|l|}{ Endpoints } \\
\hline & & & & $\mathbf{R R}(\%)$ & \begin{tabular}{|l} 
mOS \\
(months)
\end{tabular} & \begin{tabular}{|l|} 
mPFS \\
(months)
\end{tabular} \\
\hline Valle et $\mathrm{a}^{23}$ & $\begin{array}{l}\text { First-line, Phase III, } \\
\text { randomized }\end{array}$ & 410 & GEMCIS vs GEM & $\begin{array}{l}\text { DCR } 81.4 \\
\text { vs } 71.8^{*}\end{array}$ & 11.7 vs $8.1^{*}$ & 59.3 vs $42.5^{*}$ \\
\hline $\begin{array}{l}\text { JCOGIII3, FUGA- } \\
\text { BT, Morizane et } \mathrm{a}^{24}\end{array}$ & $\begin{array}{l}\text { First line, Phase III, } \\
\text { randomized }\end{array}$ & 354 & GEMCIS vs GEM+S-I & $\begin{array}{l}32.4 \text { vs } \\
29.8, \mathrm{NI}\end{array}$ & $\begin{array}{l}\text { I3.4 vs } 15.1 \text {, } \\
\mathrm{NI}\end{array}$ & 5.8 vs $6.8, \mathrm{NI}$ \\
\hline Zheng et $\mathrm{al}^{26}$ & $\begin{array}{l}\text { Second line, Phase II, } \\
\text { randomized }\end{array}$ & 60 & XELIRI vs IRI & $\begin{array}{l}13.3 \text { vs } \\
6.7\end{array}$ & 10.1 vs 7.3 & 3.7 vs $2.4^{*}$ \\
\hline Lee et $\mathrm{al}^{37}$ & $\begin{array}{l}\text { First line, Phase III, } \\
\text { randomized }\end{array}$ & 268 & GEMOX+erlotinib vs GEMOX & 30 vs $16^{*}$ & 9.5 vs 9.5 & 5.8 vs $4.2^{*}$ \\
\hline $\begin{array}{l}\text { VECTIBIL, Leone } \\
\text { et } \mathrm{al}^{38}\end{array}$ & $\begin{array}{l}\text { First line, Phase II, } \\
\text { randomized }\end{array}$ & 89 Ras WT & GEMOX+panitumumab vs GEMOX & $\begin{array}{l}26.6 \text { vs } \\
18.1\end{array}$ & 9.9 vs 10.2 & 5.3 vs 4.4 \\
\hline $\begin{array}{l}\text { TACTIC, Ferraro } \\
\text { et al }{ }^{39}\end{array}$ & $\begin{array}{l}\text { First line, Phase II, open } \\
\text { label, single arm }\end{array}$ & 78 Ras WT & GEMCIS+panitumumab & $46 \mathrm{CR} / \mathrm{RP}$ & 11.9 & 8 \\
\hline PICCA, Vogel et a ${ }^{40}$ & $\begin{array}{l}\text { First line, Phase II, open } \\
\text { label }\end{array}$ & 62 & GEMCIS+panitumumab vs GEMCIS & 45 vs 39 & 12.8 vs 20.1 & 7.6 vs 6.7 \\
\hline $\begin{array}{l}\text { BINGO } \\
\text { Malka et } a^{41}\end{array}$ & $\begin{array}{l}\text { First line, Phase II, } \\
\text { randomized }\end{array}$ & 150 & GEMOX+cetuximab vs GEMOX & 23 vs 29 & II vs 12.4 & 6 vs 5.3 \\
\hline Chen et $\mathrm{a}^{42}$ & $\begin{array}{l}\text { First line, Phase II, } \\
\text { randomized }\end{array}$ & 122 & GEMOX+cetuximab vs GEMOX & 27 vs 15 & 10.6 vs 9.8 & 6.7 vs 4.1 \\
\hline lyer et $\mathrm{al}^{43}$ & $\begin{array}{l}\text { First line, Phase II, } \\
\text { prospective }\end{array}$ & 50 & GEMCAPE+bevacizumab & 40 & 12.7 & 7 \\
\hline Larsen et $\mathrm{al}^{44}$ & $\begin{array}{l}\text { Second line, Phase II, } \\
\text { prospective }\end{array}$ & 50 & CAPE+IRI+GEM+bevacizumab & 6 & 6.4 & 3.6 \\
\hline$A B C-03$, Valle et $a^{46}$ & $\begin{array}{l}\text { First line, Phase II, } \\
\text { randomized }\end{array}$ & 124 & GEMCIS+cediranib vs GEMCIS & 44 vs 19 & 14.1 vs 11.9 & 8 vs 7.4 \\
\hline $\begin{array}{l}\text { AIO study, Moehler } \\
\text { et al }{ }^{47}\end{array}$ & $\begin{array}{l}\text { First line, Phase II, } \\
\text { randomized }\end{array}$ & 102 & GEM+sorafenib vs GEM & 8 vs 6 & 8.4 vs $I 1.2$ & 3 vs 4.9 \\
\hline Yi et al ${ }^{48}$ & $\begin{array}{l}\text { Phase II, multicenter, } \\
\text { single arm }\end{array}$ & 56 & Sunitinib & 8.9 & 12.9 & 2.4 \\
\hline $\begin{array}{l}\text { SUN-CK, Neuzillet } \\
\text { et al }{ }^{49}\end{array}$ & $\begin{array}{l}\text { Second line, Phase II, } \\
\text { single arm }\end{array}$ & 53 & Sunitinib & 15 & 9.6 & 5.2 \\
\hline $\begin{array}{l}\text { The Van Gogh study, } \\
\text { Santoro et al }{ }^{50}\end{array}$ & $\begin{array}{l}\text { First line, Phase II, } \\
\text { randomized }\end{array}$ & 173 & $\begin{array}{l}\text { Vandetanib vs Vandetanib+GEM vs } \\
\text { GEM+placebo }\end{array}$ & $\begin{array}{l}2 \text { vs } 11 \\
\text { vs } 7^{*}\end{array}$ & $\begin{array}{l}3.5 \text { vs } 3.8 \\
\text { vs } 5\end{array}$ & $\begin{array}{l}7.6 \text { vs } 9.5 \text { vs } \\
10.2\end{array}$ \\
\hline
\end{tabular}

Note: *Satistically significant.

Abbreviations: BTC, biliary tract carcinoma; CAPE, capecitabine; CIS, cisplatin; DCR, disease control rate; DFS, disease-free survival; GEM, gemcitabine; GEMCAPE, gemcitabine and capecitabine; GEMCIS, gemcitabine and cisplatin; GEMOX, gemcitabine and oxaliplatin; IRI, irinotecan; mOS, mean overall survival; mPFS, mean progressionfree survival; NI, noninferiority; OX, oxaliplatin; RFS, relapse-free survival; RR, response rate; WT, wild type; XELIRI, capecitabine and irinotecan.

\section{Second-line CT}

No established therapies after first-line failure are currently available. In this regard, a systematic review of 25 studies was performed. mOS was 7.2 months, mPFS was 3.2 months, and response rate (RR) was $7.7 \%$ with lack of evidence for recommending the use of a second-line $\mathrm{CT}$ in advanced BTC. ${ }^{25}$ A recent randomized Phase II Asian study compared irinotecan monotherapy to irinotecan and CAPE doublet (Table 2). mPFS and 9-month survival rate were better for the combination treatment ( 3.7 vs 2.4 months, $P=0.036$ and $60.9 \%$ vs $32 \%, P=0.045$, respectively). No significant prolongation of OS was registered $(P=0.107){ }^{26}$ The Phase III randomized study $\mathrm{ABC}-06$, comparing second line 5-FU and oxaliplatin (OX) to best supportive care, has recently met its recruitment target, and results are awaited. ${ }^{10}$

\section{Genetics of BTC Mutational patterns}

Increasing attention has been paid in studying the genetic alterations linked to BTC (Figure 1). Whole-exome sequencing (WES) analyses performed in different series have shown mutations with different frequencies that were correlated with different BTC sites of primary tumor. ${ }^{10}$ In a recent series, 153 BTC cases were analyzed in search for mutations in 56 genes. Most of the cases (77.1\%) harbored a driver-gene mutation and targetable pathway alterations were present in 
$68 \%$ of cases. Different sites of origin had distinct molecular profiles. KRAS mutations were the most detected ones $(28 \%$ of cases), followed by TP53 alterations (18\%). TP53 and $K R A S$ mutations were significantly associated with a worse survival and more frequent eCCAs and GBCs $(P=0.0019$ for both KRAS and TP53). Mutations in the IDH1 and IDH2 genes $(P=0.0005)$ and in the chromatin-remodeling gene $B A P 1(P=0.0097)$ were typical of iCCAs. In a recent series of KRAS wild-type (WT) BTC patients, mutated IDH1/2 was associated with a significantly higher mOS of 28.22 months compared to 11.56 months for nonmutated genotypes $(P=0.025)$. Conversely, mOS did not differ when stratification by $T P 53$ mutation was performed $(P=0.82)$.

Both the mTOR (51\% of cases) and the KRAS/NRAF/ $B R A F(34 \%)$ pathways were frequently altered. ${ }^{27} \mathrm{GBCs}$ and eCCAs were mostly associated with the APOBEC-mediated somatic molecular signature, with a high mutational burden and increased expression of the $A P O B E C 3 B$ gene. ${ }^{28}$ $A P O B E C 3 B$ is a DNA cytosine deaminase-inducing mutation in multiple human cancers. ${ }^{29}$ Differently, three main pathways linked to epigenetic regulation and oxidative phosphorylation were found altered in iCCAs: the Ras/ phosphatidylinositol-4,5-bisphosphate 3-kinase pathway, the $p 53$ cell cycle signaling, and $T G F-\beta /$ Smad signaling. ${ }^{30}$ FGFR2 fusions were reported as the most frequent targetable alteration in iCCAs, with a prevalence of $45 \%$ described in a recent series. ${ }^{31} \mathrm{~A}$ WES analysis performed on GBCs only showed mutations in the ERBB family of proteins and downstream genes in the $35.8 \%$ of cases, with a significant negative prognostic role at multivariate analysis $(P=0.001){ }^{32}$ $H E R-2$ overexpression was reported with a higher prevalence in GBCs as well $(19 \%){ }^{33}$

Other relevant deregulated pathways in BTCs are the VEGF-VEGFR-1/2 (with a VEGF overexpression in more than half cases of both iCCAs and eCCAs) and the EGFR pathway, with an overexpression in $27.4 \%$ of iCCAs and $19.2 \%$ of eCCAs. These pathways, when altered, were associated with tumor progression, recurrence, and hematogenic metastatic spread in iCCAs and eCCAs. ${ }^{34}$

The $W N T / \beta$-catenin pathway is upregulated in hilar CCA. ${ }^{35}$ cMET overexpression was seen in $11.7 \%$ of iCCAs and $16.2 \%$ of eCCAs, as well. ${ }^{36}$ Higher $c$-MET expression

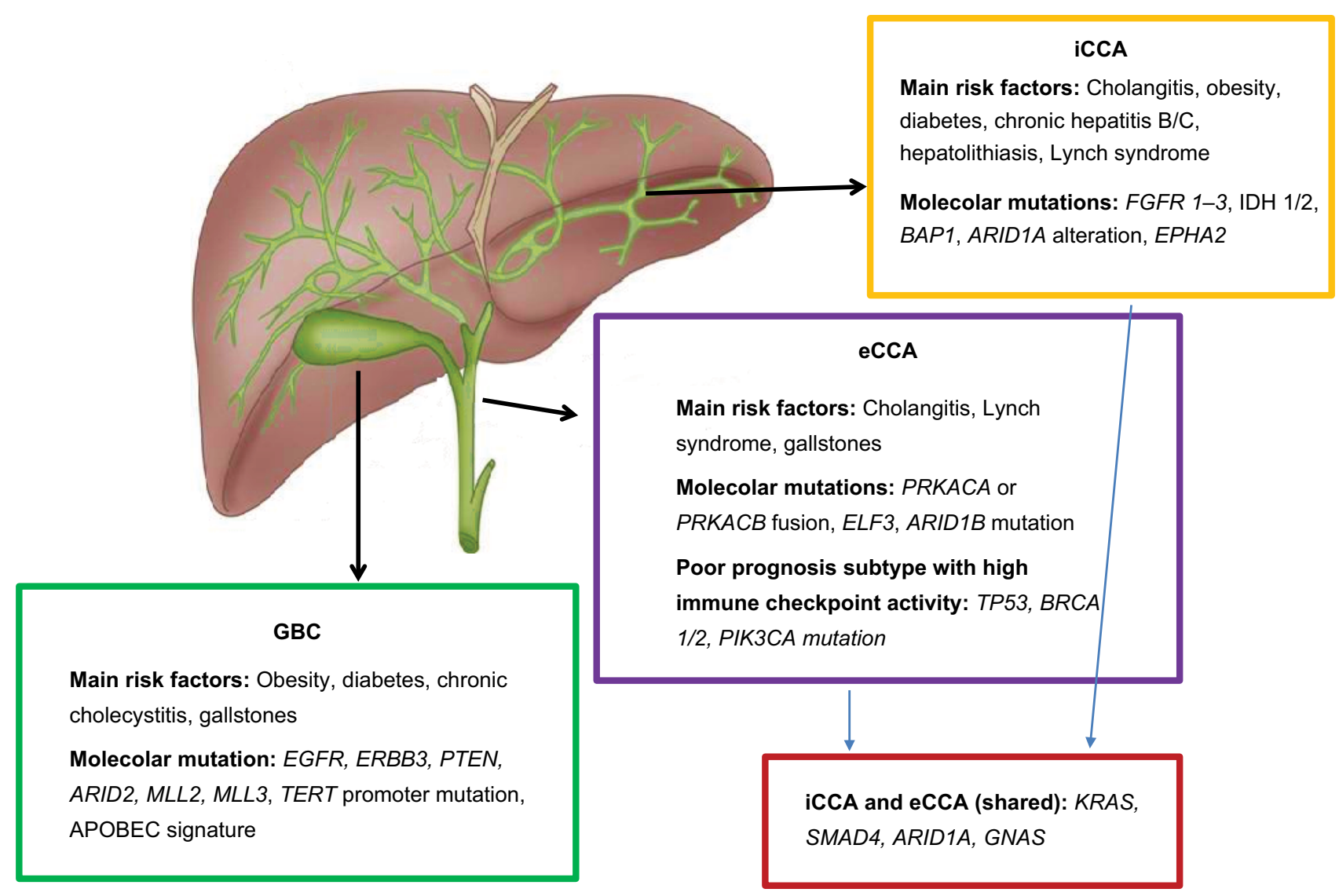

Figure I Risk factors and molecular alterations of BTC.

Abbreviations: BTC, biliary tract carcinoma; eCCA, extrahepatic cholangiocarcinoma; GBC, gallbladder cancer; iCCA, intrahepatic cholangiocarcinoma. 
was associated with poor 5-year survival rate for both CCAs overall $(P=0.0046)$ and iCCAs $(P=0.0013) .{ }^{36}$

In conclusion, several genetic alterations are associated with BTCs representing possible targets of antitumor biologic agents. Among them, KRAS, TP53, IDH, and BAPl mutations and FGFR2 fusions are the most frequent ones.

\section{Clinical studies with targeted therapies}

Several Phase II and III trials in first- and second-line treatment of advanced BTCs have been performed so far. Both targeted therapies and combinations of $\mathrm{CT}$ and biological agents have been tested (Table 2).

Results using different anti-EGFR agents have been disappointing. A Phase III randomized trial compared combination therapy with GEMOX with or without the addition of erlotinib. The experimental arm had a higher number of objective responses ( 40 vs 21 patients, $P=0.005$ ) but no difference in OS was registered $(P=0.611) .{ }^{37}$ Two Phase II trials tested panitumumab in BTC patients with KRAS WT genotype. The randomized Phase II Vecti-BIL study randomized subjects to GEMOX doublet or the same combination with panitumumab. No differences in $\operatorname{mOS}(P=0.42)$ and PFS $(P=0.27)$ were described. ${ }^{38}$ Similarly, the Phase II TACTIC study tested the combination of GEMCIS, and panitumumab in BTC KRAS WT patients. This single-arm trial showed a good tolerability and a promising clinical efficacy, with $46 \%$ of patients having complete or partial response, mPFS of 8 months, and OS of 11.9 months. ${ }^{39} \mathrm{~A}$ similar Phase II randomized study ("PICCA" study) planned a 2:1 randomization between GEMCIS CT and the doublet combined with panitumumab. KRAS WT patients only were included. PFS rate at 6 months was $54 \%$ in patients treated with the doublet plus panitumumab vs $73 \%$ for patients treated with CT alone. mOS was 12.8 months for CT and panitumumab vs 20.1 months for CT alone. ${ }^{40}$ Additionally, the Phase II randomized BINGO trial tested GEMOX with or without cetuximab in patients unselected for $K R A S$ status. mPFS was 6.1 in the combination arm vs 5.5 months in the CT group. mOS was 11 months with cetuximab and 12.4 months in the CT alone group. Thus, the addition of cetuximab did not demonstrate to enhance the activity of CT alone. ${ }^{41}$ A study with a similar design, but with stratification based on $K R A S$ status, found a trend toward a better mPFS with the addition of cetuximab (6.7 vs 4.1 months, $P=0.05)$ but not mOS (10.6 vs 9.8 months, $P=0.91){ }^{42}$

A recent meta-analysis of 12 studies did not reveal a survival advantage for the addition of anti-EGFR agents to combination CT, with an mPFS for the experimental arm of
7.6 vs 6.7 months for CT alone and a mOS of 12.6 vs 11.6 months, respectively. ${ }^{40}$

Some studies in BTC tested angiogenesis inhibition with bevacizumab and cediranib. In a recent series, bevacizumab was added to CT with gemcitabine and capecitabine (GEMCAPE) in a Phase II design. Survival outcomes were comparable to historical controls from the ABC-02 trial, with an mPFS of 8.1 months and OS of 10.2 months. Therefore, the addition of bevacizumab was not shown to improve outcome as compared with standard $\mathrm{CT}^{43}$ As second-line treatment together with CAPE, irinotecan, and GEM, bevacizumab showed a limited benefit, with an mPFS of 3.6 months, mOS of 6.4 months, and RR of $6 \% .{ }^{44}$

Ramucirumab, a monoclonal antibody targeting VEGFR2, showed an mPFS of 2.73 months and OS of 6.31 months when administered in advanced and pretreated BTC. In a minority of patients (13\%), mPFS was superior to 24 weeks. These results, which are comparable to those obtained with more toxic CT regimens, may require further investigations in the field of refractory BTC. ${ }^{45}$

Cediranib, an oral inhibitor of the VEGF pathway, was tested in the randomized Phase II ABC-03 trial. In the study design, the experimental arm included standard CT with GEMCIS with the addition of cediranib, while the control arm included CT only. The addition of cediranib did not improve mPFS (8.0 vs 7.4 months for control, $P=0.72$ ) and resulted in higher toxicity, hypertension, diarrhea, and bone marrow toxicity. ${ }^{46}$ Among other oral multi-kinase inhibitors, sorafenib, sunitinib, and vandetanib have been used in BTC trials. A double-blind, placebo-controlled Phase II study compared GEM and sorafenib vs GEM alone. mPFS and OS were similar between the two arms $(P=0.859$ and $P=0.775$, respectively). However, a significant difference in OS was present when patients developed liver metastases after resection of primary BTC and were treated with sorafenib compared to placebo $(P=0.019) .{ }^{47}$ Sunitinib was used in second line in a multicentre Asian study. Time to progression was 1.7 months, and the efficacy of the oral drug was poor with an objective RR (ORR) of $8.9 \%$ and a disease control rate (DCR) of 50\%. ${ }^{48}$ In a second trial (SUN-CK Phase II), sunitinib was used in the second-line treatment of iCCAs after progression to GEM. Results were promising with mOS of 9.6 months, while PFS was 5.2 months. DCR was $85 \%$. ${ }^{49}$ Vandetanib, an oral inhibitor of VEGFR2, EGFR/HER1, and RET, was tested in subjects with advanced BTC in the "Van Gogh" study. Patients were randomized between three different arms: vandetanib alone, vandetanib plus GEM, and GEM plus placebo. PFS was not significantly different among 
the three different arms $(P=0.18)$. Similarly, no difference in mOS was observed $(P=0.066){ }^{50}$

Selumetinib, an oral $M E K$ inhibitor acting in the $R A S /$ $R A F / M E K / E R K$ signaling pathway, was administered together with GEMCIS in a Phase II trial. Efficacy was poor with no difference between the two selumetinib and GEMCIS arms (with different doses of selumetinib) and the control arm represented by standard CT $(P=0.37$ for selumetinib $75 \mathrm{mg}$ twice a day continuously and 0.53 for doses on days $1-5$ and 8-19 every 21 days). Addition of CT was detrimental adding toxicities and leading to a lower selumetinib dose intensity. ${ }^{51}$ A single-arm, Phase II and biomarker study of cabozantinib was performed for patients with advanced refractory CCA. Cabozantinib is a multi-kinase inhibitor with potent activity against VEGFR2 and MET. Previously treated patients with unresectable or metastatic CCA received cabozantinib (60 mg orally and daily on a continuous schedule). In this trial, cabozantinib demonstrated limited activity and significant toxicity. mPFS was 1.8 months, and mOS was 5.2 months. ${ }^{52}$

Another Japanese trial investigated efficacy and safety of trametinib in Japanese patients with advanced BTC refractory to GEM-based therapy. In this Phase IIa open-label, single-arm trial, patients received oral trametinib $2 \mathrm{mg}$ once daily until progressive disease, death, or unacceptable toxicity. The primary endpoint was to determine the 12-week nonprogression (PD) rate. Secondary assessments included safety, mPFS, mOS, and overall response rate. The non-PD rate at week 12 was $10 \%$. mPFS was 10.6 weeks. ${ }^{53}$

Another tested combination was pazopanib (a VEGFR tyrosine kinase inhibitor) and trametinib (MEK inhibitor). In this open-label, multicentre, single-arm trial, adults with advanced unresectable CCA received pazopanib $800 \mathrm{mg}$ daily and trametinib $2 \mathrm{mg}$ daily until disease progression or unacceptable toxicity. mPFS was 3.6 months, mOS was 6.4 months, ORR was 5\%, and DCR was 75\%. Despite an acceptable toxicity and evidence of clinical activity, the combination did not achieve a statistically significant improvement in 4-month PFS. ${ }^{54}$

All in all, targeted therapies failed to prolong survival outcomes in the treatment of nonresectable and metastatic BTC, both in first- and second-line settings. Combination CT still remains the standard of care in advanced disease.

\section{Future prospects}

\section{Immunotherapy}

BTC represents a potentially attractive subset for immunebased therapies because of its association with chronic inflammation. ${ }^{55}$ In a retrospective series of resected BTC, a low expression of cytotoxic T-lymphocyte antigen-4 (CTLA-4) in the peritumoral tissue of resected BTC was associated with a higher survival than a higher expression $(P=0.02)$. Therefore, CTLA-4 had a prognostic role, reflecting the capacity of the immune system to react against the tumor. CTLA-4 is expressed on the surface of regulatory $\mathrm{T}$ cells (Tregs) and binds to CD80 on antigen-presenting cells with inhibitory effects on cytotoxic cells. In patients receiving AT, strong CD80 expression was associated with a shorter survival $(P=0.02)$. A possible explanation for that is given by the enrichment of activated Tregs in the tumoral microenvironment, inhibiting the response to $\mathrm{CT} .{ }^{13} \mathrm{BTC}$ with a high mutational load and with elevated expression of CTLA-4 and PD-L1 was shown to have the worst prognosis. ${ }^{28}$ Recently, a correlation between PD-L1 expression by either neoplastic $(P=0.01)$ or inflammatory cell aggregates $(P=0.005)$ and a high density of CD3-positive tumor infiltrating lymphocytes was reported, and in addition, the number of PD-L1 inflammatory cell aggregates was superior in case of a high PD-1 expression $(P<0.0001) .{ }^{56}$ Interim results of the KEYNOTE-028 trial, a Phase Ib trial of pembrolizumab in advanced PD-L1-positive BTC progressing after a first-line therapy, showed a $17 \%$ ORR with $17 \%$ of partial response, $17 \%$ of stable disease, $52 \%$ of progressive disease, and a good profile of tolerability. ${ }^{57}$ Following this experience, a Phase I study of 5-FU and oxaliplatin (FOLFOX-6) CT in combination with pembrolizumab with an expanded Phase II cohort in BTC was planned, and such a trial is ongoing (NCT02268825). A Phase I trial assessed safety and efficacy of ramucirumab (VEGFR2 antagonist) with pembrolizumab (PD-1 antagonist) in biomarker unselected patients with previously treated advanced or metastatic BTC. The primary endpoint was safety and tolerability of the combination. Secondary endpoints included ORR. Ramucirumab-pembrolizumab combination showed limited clinical activity with infrequent grade 3-4 treatment-related adverse events (hypertension and neutropenia); ORR was $4 \%$, mPFS was 1.6 months, and mOS was 6.4 months. ${ }^{58}$

The anti PD-1 nivolumab is currently being tested in a Phase II, two-arm study with a randomization between the combination of nivolumab and GEMCIS vs a dual immunotherapy with nivolumab and ipilimumab. The primary endpoint was the PFS rate at 6 months, with an accrual goal of 32 patients per arm. ${ }^{59}$ Another early phase trial is ongoing using nivolumab therapy together with entinostat, a histone deacetylase inhibitor. In murine models of pancreatic cancer, entinostat was shown to inhibit suppressive myeloid-derived suppressor cells (MDSC) with a consequent recruitment in T 
cells and a synergistic effect together with anti-PD-1 therapy in order to improve survival. The Phase I trial will investigate the antitumor activity of the combination (measured as ORR) in unresectable or advanced pancreatic cancer and BTC patients who have progressed after at least one line of therapy. ${ }^{60}$

\section{Targeted therapies}

Several studies are ongoing with agents targeting $I D H 1 / 2$ mutations. Specifically, the AG120 IDH1 inhibitor is currently being tested at the dose of $500 \mathrm{mg}$ once daily (expansion phase) after the promising initial results showed no dose-limiting toxicities (NCT02073994). With a highthroughput drug screen of 17 iCCAs cases, the multityrosine kinase inhibitor (TKI) dasatinib was shown to be active in tumors harboring $I D H$ mutations. ${ }^{61}$ On that basis, a Phase II trial of dasatinib in patients with $I D H$-mutant advanced iCCAs is ongoing (NCT02428855).

Another target of actual interest is FGFR. The ongoing Phase II trial with the oral FGFR inhibitor infigratinib in advanced CCA with FGFR aberrations (gene fusions, translocations, or other genetic alterations) showed interesting results with an overall response rate of $14.8 \%(18.8 \%$ for FGFR2 fusions only). ${ }^{62}$ Other inhibitors, such as the irreversible FGFR inhibitor TAS-120 (NCT02052778) and the non-selective TKI ARQ-087, targeting FGFR 1-3 (NCT01752920), are being tested in Phase I and II trials, respectively. ${ }^{10}$

As far as anti-angiogenic agents are concerned, a Phase II trial of GEM and pazopanib combination as first-line treatment of advanced BTC is ongoing (NCT01855724). A further Phase II trial involves regorafenib as single agent in pretreated BTC (NCT02053376). Recently, a French randomized Phase II study reported data on the activity of regorafenib in combination with GEMOX in advanced BTC. Regorafenib showed acceptable tolerability when combined with GEMOX with toxicities mainly imputable to conventional CT. ${ }^{63} \mathrm{~A}$ randomized Phase II double-blind trial tested the combination of ramucirumab or merestinib (MET inhibitor) or placebo in addition to GEMCIS. ${ }^{64}$

$P I 3 K-A K T-m T O R$ pathway is still being investigated in BTC. Copanlisib, a PI3K inhibitor, is under evaluation together with GEMCIS in advanced CCA (NCT02631590).

\section{Conclusion}

Treatment of CCA is challenging both in early and advanced settings. Surgery is the only potentially curative option, but even in case of radical resection, relapses are common.
Adjuvant CAPE has been shown to significantly improve outcomes and should be always offered after surgery. Outcomes of metastatic disease remain poor with very few advances achieved over the last years. To date, combination CT (for example, GEMCIS) still represents the best palliative treatment option. The addition of targeted agents to CT failed to demonstrate any further benefit. The increasing knowledge of molecular mechanisms underlying the tumoral processes, along with the availability of more and more accessible and comprehensive diagnostic techniques, will hopefully allow to identify reliable biomarkers able to guide the most effective treatments in the near future.

\section{Disclosure}

The authors report no conflicts of interest in this work.

\section{References}

1. Valle JW. Advances in the treatment of metastatic or unresectable biliary tract cancer. Ann Oncol. 2010;21(Suppl 7):vii345-vii348.

2. Kim SJ, Akita M, Sung YN, et al. MDM2 Amplification in intrahepatic cholangiocarcinomas: its relationship with large-duct type morphology and uncommon KRAS mutations. Am J Surg Pathol. 2018;42(4):512-521.

3. GLOBOCAN [homepage on the Internet]. 2012 v1.0, Cancer Incidence and Mortality Worldwide: IARC Cancer Base No. 11. International Agency for Research on Cancer; 2013. Available from: http://globocan. iarc.fr. Accessed August 1, 2018.

4. Shaib Y, El-Serag HB. The epidemiology of cholangiocarcinoma. Semin Liver Dis. 2004;24(2):115-125.

5. Hundal R, Shaffer EA. Gallbladder cancer: epidemiology and outcome. Clin Epidemiol. 2014;6:99-109.

6. Harrington J, Carter L, Basu B, Cook N. Drug development and clinical trial design in pancreatico-biliary malignancies. Curr Probl Cancer. 2018;42(1):73-94.

7. Horgan AM, Amir E, Walter T, Knox JJ. Adjuvant therapy in the treatment of biliary tract cancer: a systematic review and meta-analysis. $J$ Clin Oncol. 2012;30(16):1934-1940.

8. Endo I, Gonen M, Yopp AC, et al. Intrahepatic cholangiocarcinoma: rising frequency, improved survival, and determinants of outcome after resection. Ann Surg. 2008;248(1):84-96.

9. Valle JW, Borbath I, Khan SA, et al. Biliary cancer: ESMO Clinical Practice Guidelines for diagnosis, treatment and follow-up. Ann Oncol. 2016;27(Suppl 5):v28-v37.

10. Valle JW, Lamarca A, Goyal L, Barriuso J, Zhu AX. New horizons for precision medicine in biliary tract cancers. Cancer Discov. 2017;7(9):943-962.

11. Squadroni M, Tondulli L, Gatta G, Mosconi S, Beretta G, Labianca R. Cholangiocarcinoma. Crit Rev Oncol Hematol. 2017;116:11-31.

12. Takada T, Amano H, Yasuda $\mathrm{H}$, et al. Is postoperative adjuvant chemotherapy useful for gallbladder carcinoma? A phase III multicenter prospective randomized controlled trial in patients with resected pancreaticobiliary carcinoma. Cancer. 2002;95(8):1685-1695.

13. Ghidini M, Tomasello G, Botticelli A, et al. Adjuvant chemotherapy for resected biliary tract cancers: a systematic review and meta-analysis. HPB (Oxford). 2017;19(9):741-748.

14. Ebata T, Hirano S, Konishi M, et al. Randomized clinical trial of adjuvant gemcitabine chemotherapy versus observation in resected bile duct cancer. Br J Surg. 2018;105(3):192-202.

15. Edeline J, Bonnetain F, Phelip JM, et al. Gemox versus surveillance following surgery of localized biliary tract cancer: results of the PRODIGE 12-ACCORD 18 (UNICANCER GI) phase III trial. J Clin Oncol. 2017;35(4 Suppl):225. 
16. Primrose JN, Fox R, Palmer DH, et al. Adjuvant capecitabine for biliary tract cancer: the BILCAP randomized study. J Clin Oncol. 2017;35(15 Suppl):4006

17. Bridgewater J, Fox R, Primrose J, Group TBS. Exploratory analyses of the BILCAP study. 2018 ASCO Annual Meeting; 2018; Chicago.

18. Stein A, Arnold D, Bridgewater J, et al. Adjuvant chemotherapy with gemcitabine and cisplatin compared to observation after curative intent resection of cholangiocarcinoma and muscle invasive gallbladder carcinoma (ACTICCA-1 trial): a randomized, multidisciplinary, multinational phase III trial. BMC Cancer. 2015;15:564.

19. Phelip JM, Vendrely V, Rostain F, et al. Gemcitabine plus cisplatin versus chemoradiotherapy in locally advanced biliary tract cancer: Fédération Francophone de Cancérologie Digestive 9902 phase II randomised study. Eur J Cancer. 2014;50(17):2975-2982.

20. Tao R, Krishnan S, Bhosale PR, et al. Ablative radiotherapy doses lead to a substantial prolongation of survival in patients with inoperable intrahepatic cholangiocarcinoma: a retrospective dose response analysis. J Clin Oncol. 2016;34(3):219-226.

21. Edeline J, Du FL, Rayar M, et al. Glass microspheres $90 \mathrm{Y}$ selective internal radiation therapy and chemotherapy as first-line treatment of intrahepatic cholangiocarcinoma. Clin Nucl Med.2015;40(11):851-855.

22. Al-Adra DP, Gill RS, Axford SJ, Shi X, Kneteman N, Liau SS. Treatment of unresectable intrahepatic cholangiocarcinoma with yttrium-90 radioembolization: a systematic review and pooled analysis. Eur J Surg Oncol. 2015;41(1):120-127.

23. Valle J, Wasan H, Palmer DH, et al. Cisplatin plus gemcitabine versus gemcitabine for biliary tract cancer. $N \mathrm{Engl} \mathrm{J} \mathrm{Med.}$ 2010;362(14):1273-1281.

24. Morizane C, Okusaka T, Mizusawa J. Randomized phase III study of gemcitabine plus S-1 combination therapy versus gemcitabine plus cisplatin combination therapy in advanced biliary tract cancer: a Japan Clinical Oncology Group study (JCOG1113, FUGA-BT). J Clin Oncol. 2018;36(4 Suppl):205-205.

25. Lamarca A, Hubner RA, David Ryder W, Valle JW. Second-line chemotherapy in advanced biliary cancer: a systematic review. Ann Oncol. 2014;25(12):2328-2338

26. Zheng Y, Tu X, Zhao P, et al. A randomised phase II study of second-line XELIRI regimen versus irinotecan monotherapy in advanced biliary tract cancer patients progressed on gemcitabine and cisplatin. $\mathrm{Br} J$ Cancer. 2018;119(3):291-295.

27. Simbolo M, Fassan M, Ruzzenente A, et al. Multigene mutational profiling of cholangiocarcinomas identifies actionable molecular subgroups. Oncotarget. 2014;5(9):2839-2852.

28. Nakamura H, Arai Y, Totoki Y, et al. Genomic spectra of biliary tract cancer. Nat Genet. 2015;47(9):1003-1010.

29. Zou J, Wang C, Ma X, Wang E, Peng G. APOBEC3B, a molecular driver of mutagenesis in human cancers. Cell Biosci. 2017;7:29.

30. Zou S, Li J, Zhou H, et al. Mutational landscape of intrahepatic cholangiocarcinoma. Nat Commun. 2014;5:5696.

31. Sia D, Losic B, Moeini A, et al. Massive parallel sequencing uncovers actionable FGFR2-PPHLN1 fusion and ARAF mutations in intrahepatic cholangiocarcinoma. Nat Commun. 2015;6:6087.

32. Li M, Zhang Z, Li X, et al. Whole-exome and targeted gene sequencing of gallbladder carcinoma identifies recurrent mutations in the ErbB pathway. Nat Genet. 2014;46(8):872-876.

33. Galdy S, Lamarca A, Mcnamara MG, et al. HER2/HER3 pathway in biliary tract malignancies; systematic review and meta-analysis: a potential therapeutic target? Cancer Metastasis Rev. 2017;36(1):141-157.

34. Yoshikawa D, Ojima H, Iwasaki M, et al. Clinicopathological and prognostic significance of EGFR, VEGF, and HER2 expression in cholangiocarcinoma. Br J Cancer. 2008;98(2):418-425.

35. Chen W, Liang J, Huang L, et al. Characterizing the activation of the Wnt signaling pathway in hilar cholangiocarcinoma using a tissue microarray approach. Eur J Histochem. 2016;60(1):2536.

36. Miyamoto M, Ojima H, Iwasaki M, et al. Prognostic significance of overexpression of c-Met oncoprotein in cholangiocarcinoma. $\mathrm{Br} \mathrm{J}$ Cancer. 2011;105(1):131-138.
37. Lee J, Park SH, Chang HM, et al. Gemcitabine and oxaliplatin with or without erlotinib in advanced biliary-tract cancer: a multicentre, openlabel, randomised, phase 3 study. Lancet Oncol. 2012;13(2):181-188.

38. Leone F, Marino D, Cereda S, et al. Panitumumab in combination with gemcitabine and oxaliplatin does not prolong survival in wild-type KRAS advanced biliary tract cancer: a randomized phase 2 trial (VectiBIL study). Cancer. 2016;122(4):574-581.

39. Ferraro D, Goldstein D, O'Connell RL, et al. TACTIC: a multicentre, open-label, single-arm phase II trial of panitumumab, cisplatin, and gemcitabine in biliary tract cancer. Cancer Chemother Pharmacol. 2016;78(2):361-367.

40. Vogel A, Kasper S, Bitzer M, et al. PICCA study: panitumumab in combination with cisplatin/gemcitabine chemotherapy in KRAS wildtype patients with biliary cancer-a randomised biomarker-driven clinical phase II AIO study. Eur J Cancer. 2018;92:11-19.

41. Malka D, Cervera P, Foulon S, et al. Gemcitabine and oxaliplatin with or without cetuximab in advanced biliary-tract cancer (BINGO): a randomised, open-label, non-comparative phase 2 trial. Lancet Oncol. 2014;15(8):819-828.

42. Chen JS, Hsu C, Chiang NJ, et al. A KRAS mutation status-stratified randomized phase II trial of gemcitabine and oxaliplatin alone or in combination with cetuximab in advanced biliary tract cancer. Ann Oncol. 2015;26(5):943-949.

43. Iyer RV, Pokuri VK, Groman A, et al. A multicenter Phase II study of gemcitabine, capecitabine, and bevacizumab for locally advanced or metastatic biliary tract cancer. Am J Clin Oncol. 2018;41(7): 649-655.

44. Larsen FO, Markussen A, Diness LV, Nielsen D. Efficacy and safety of capecitabine, irinotecan, gemcitabine, and bevacizumab as second-line treatment in advanced biliary tract cancer: a Phase II cancer: A Phase II Study. Oncology. 2018;94(1):19-24.

45. Mizrahi J, Javle MM, Xiao L, et al. A phase II study of ramucirumab for advanced, pre-treated biliary cancers. 2018 ASCO Annual Meeting; 2018; Chicago.

46. Valle JW, Wasan H, Lopes A, et al. Cediranib or placebo in combination with cisplatin and gemcitabine chemotherapy for patients with advanced biliary tract cancer (ABC-03): a randomised phase 2 trial. Lancet Oncol. 2015;16(8):967-978.

47. Moehler M, Maderer A, Schimanski C, et al. Gemcitabine plus sorafenib versus gemcitabine alone in advanced biliary tract cancer: a double-blind placebo-controlled multicentre phase II AIO study with biomarker and serum programme. Eur J Cancer. 2014;50(18):3125-3135.

48. Yi JH, Thongprasert S, Lee J, et al. A phase II study of sunitinib as a second-line treatment in advanced biliary tract carcinoma: a multicentre, multinational study. Eur J Cancer. 2012;48(2):196-201.

49. Neuzillet C, Seitz JF, Fartoux L, et al. Sunitinib as second-line treatment in patients with advanced intrahepatic cholangiocarcinoma (SUN-CK phase II trial): safety, efficacy, and updated translational results. J Clin Oncol. 2015;33(3 Suppl):343-343.

50. Santoro A, Gebbia V, Pressiani T, et al. A randomized, multicenter, phase II study of vandetanib monotherapy versus vandetanib in combination with gemcitabine versus gemcitabine plus placebo in subjects with advanced biliary tract cancer: the VanGogh study. Ann Oncol. 2015;26(3):542-547.

51. Doherty M, Tam VC, Mcnamara MG, et al. Selumetinib (Sel) and cisplatin/gemcitabine (CisGem) for advanced biliary tract cancer (BTC): a randomized trial. J Clin Oncol. 2018;36(15 Suppl):4084-4084.

52. Goyal L, Zheng H, Yurgelun MB, et al. A phase 2 and biomarker study of cabozantinib in patients with advanced cholangiocarcinoma. Cancer. 2017;123(11):1979-1988.

53. Ikeda M, Ioka T, Fukutomi A, et al. Efficacy and safety of trametinib in Japanese patients with advanced biliary tract cancers refractory to gemcitabine. Cancer Sci. 2018;109(1):215-224.

54. Shroff RT, Yarchoan M, O'Connor A, et al. The oral VEGF receptor tyrosine kinase inhibitor pazopanib in combination with the MEK inhibitor trametinib in advanced cholangiocarcinoma. Br J Cancer. 2017;116(11):1402-1407. 
55. Goldstein D, Lemech C, Valle J. New molecular and immunotherapeutic approaches in biliary cancer. ESMO Open. 2017;2(Suppl 1): e000152.

56. Fontugne J, Augustin J, Pujals A, et al. PD-L1 expression in perihilar and intrahepatic cholangiocarcinoma. Oncotarget. 2017;8(15):24644-24651.

57. Bang YJ, Doi T, Braud FD, et al. 525 Safety and efficacy of pembrolizumab (MK-3475) in patients (pts) with advanced biliary tract cancer: Interim results of KEYNOTE-028. Eur J Cancer. 2015;51:S112.

58. Arkenau HT, Martin-Liberal J, Calvo E, et al. Ramucirumab plus pembrolizumab in patients with previously treated advanced or metastatic biliary tract cancer: nonrandomized, open-label, phase I trial (JVDF). Oncologist. 2018:theoncologist.2018-0044.

59. Sahai V, Griffith KA, Beg MS, Zalupski M. A multi-center randomized phase II study of nivolumab in combination with gemcitabine/ cisplatin or ipilimumab as first line therapy for patients with advanced unresectable biliary tract cancer. J Clin Oncol. 2018;36(15 Suppl):TPS4142-TPS541.
60. Baretti M, Durham JN, Walker R, et al. Entinostat in combination with nivolumab for patients with advanced cholangiocarcinoma and pancreatic adenocarcinoma. 2018 Asco Annual Meeting; 2018; Chicago.

61. Saha SK, Gordan JD, Kleinstiver BP, et al. Isocitrate dehydrogenase mutations confer dasatinib hypersensitivity and SRC dependence in intrahepatic cholangiocarcinoma. Cancer Discov. 2016;6(7):727-739.

62. Javle M, Lowery M, Shroff RT, et al. Phase II study of BGJ398 in patients with FGFR-altered advanced cholangiocarcinoma. J Clin Oncol. 2018;36(3):276-282.

63. Assenat E, Guimbaud R, Fraisse J, et al. A multicenter phase Ib-IIR trial assessing the activity of regorafenib combined with modified gemcitabine: oxaliplatin (mGEMOX) in patients with advanced biliary tract cancer (aBTC). J Clin Oncol. 2018;36(15 Suppl):e16134-e16134.

64. Sama AR, Denlinger CS, Vogel A, et al. Gemcitabine and cisplatin plus ramucirumab or merestinib or placebo in first-line treatment for advanced or metastatic biliary tract cancer: a double-blind, randomized phase II trial. J Clin Oncol. 2017;35(4 Suppl):TPS509-TPS509.
Cancer Management and Research

\section{Publish your work in this journal}

Cancer Management and Research is an international, peer-reviewed open access journal focusing on cancer research and the optimal use of preventative and integrated treatment interventions to achieve improved outcomes, enhanced survival and quality of life for the cancer patient. The manuscript management system is completely online and includes

\section{Dovepress}

a very quick and fair peer-review system, which is all easy to use. Visit http://www.dovepress.com/testimonials.php to read real quotes from published authors. 